\title{
Migration, Integration und Isolation. Zielgruppenspezifische Fallanalyse der Lebensbereiche Familie, Bildung und Beruf weiblicher Migrantinnen in Tirol
}

\author{
Laura Volgger \\ Kerngebiet: Zeitgeschichte \\ eingereicht bei: assoz. Prof. Mag. Dr. Eva Pfanzelter (MA) \\ eingereicht im: WiSe 2017/18 \\ Rubrik: SE-Arbeit
}

\begin{abstract}
Migration, Integration, Isolation. Analysis of the areas family, education and labour market integration of female migrants in Tyrol

The connection between migration, integration and isolation is currently being discussed in political, psychological and social sciences as well as the area of healthcare. The purpose of this paper is to analyse the social group of female migrants with small children within the categories family, education and labour market integration. It shows the importance of an intensified integration of this specific group that is highly affected by social isolation through lack of educational and labour market integration. Whereas the first part outlines important theories of integration and assimilation, the empirical part allows a more individual consideration of female migrants and may direct future research on the connection of family, education and labour market integration and their impact on other social minority groups.
\end{abstract}

\section{Einleitung}

Die europäische Migrations-, Asyl- und Integrationspolitik unterliegt ständigen Veränderungen. Globalisierung, Internationalisierung und die aktuell steigende Anzahl von Einwandernden stellen eine große Herausforderung sowohl für die Europäische Union, die einzelnen Mitgliedstaaten, als auch die Regionen und Kommunen dar. Fehlende 
gesamteuropäische Richtlinien sowie die Fragmentierung1 der Migrations-, Asyl- und Integrationspolitik, begünstigt durch die mangelnde Solidarität ${ }^{2}$ vieler Mitgliedstaaten, rücken die Kommunen als Orte des direkten Geschehens von Migration und Integration ins Blickfeld. Diese Kommunen, worunter Städte oder Gemeinden verstanden werden, können Migrationspolitik im engeren Sinne zwar nur erschwert kontrollieren. ${ }^{3}$ Die Integration ${ }^{4}$ von Migrant*innen fällt im Sinne des altbekannten Topos im Integrationsdiskurs, dass Integration, vor Ort' stattfindet, allerdings in ihren Zuständigkeitsbereich. Somit ist die Integration ethnischer Minderheiten mittlerweile eine der wichtigsten aktuellen gesellschaftspolitischen Herausforderungen im Zusammenhang mit Überlegungen, wie man Zuwanderung dauerhaft steuern kann. ${ }^{5}$ Dabei gilt festzuhalten, dass sich die österreichische Integrationspolitik in den letzten zehn Jahren im Gegensatz zu den in der zweiten Hälfte des 20. Jahrhunderts betriebenen Anwerbungen ausländischer Arbeitskräfte im rechtlichen und sozialen Bereich positiv verändert hat. Trotzdem sind Migrations- und Integrationspolitik besonders in Österreich immer noch vielfach auf die Abwehr des Fremden ausgerichtet. ${ }^{6}$

Vor dem Hintergrund dieser Überlegungen beschäftigt sich der vorliegende Aufsatz mit dem Thema der Integration auf kommunaler Ebene, wobei der Fokus auf die Tiroler Landeshauptstadt Innsbruck in Österreich gelegt wird. Die in den letzten Jahren auffallend aktiv gewordene kommunale und regionale Integrationspolitik führte zur Entstehung zahlreicher Projekte, Bildungs- und Beratungseinrichtungen für Zugewanderte und Geflüchtete. Neben den Angebotsbereichen wie Deutschkursen, Gesundheit und Soziales, Wohnen oder Bildung und Beruf wurden zielgruppenspezifische Angebote für Frauen geschaffen, so etwa die Vereine Frauen im Brennpunkt (Frauenberatung und Kinderbetreuung), DOWAS (Beratungsstelle für Wohnen) oder Frauen aus allen Ländern (Bildungs- und Beratungseinrichtung). Dies ist für den vorliegenden Beitrag

1 Steffen Angenendt/Anne Koch, Global Migration Governance im Zeitalter gemischter Wanderungen. Folgerungen für eine entwicklungsorientierte Migrationspolitik, in: SWP-Studien 8 (2017), S. 1-34.

2 Raphael Bossong, Zwänge und Spielräume für eine europäische Grenz- und Flüchtlingspolitik. Kurzfristige Szenarien, in: Stiftung Wissenschaft und Politik 38 (2017), S. 1-4; Boldizsár Nagy, Sharing the Responsibility or Shifting the Focus? The Responses of the EU and the Visegrad Countries to the Post-2015 Arrival of Migrants and Refugees, in: Global Turkey in Europe 17 (2017), S. 1-20.

3 Bommes diskutiert in seinem Beitrag die strukturelle Stellung der Kommunen in Integrationsfragen. Er belegt, dass die Kommunen zwar die breitere Migrationspolitik nicht beeinflussen können, allerdings global denken müssen, um lokal handeln zu können. Michael Bommes, Die Rolle der Kommunen in der bundesdeutschen Migrations- und Integrationspolitik, in: Frank Gesemann/Roland Roth (Hrsg.), Handbuch Lokale Integrationspolitik, Wiesbaden 2018, S. 99-123.

4 Der Begriff stützt sich auf die von Esser beschriebene Definition, wonach „Integration“ den „Zusammenhalt von Teilen in einem ,systemischen' Ganzen“ verstanden wird. Hartmut Esser, Integration und ethnische Schichtung (Mannheimer Zentrum für Europäische Sozialforschung, Arbeitspapiere 40), Mannheim 2001, S. 1.

5 Während die Thematisierung von Integrationserfordernissen den Migrationsdiskurs seit den 1970ern begleitet hat, wurde die Integration erst durch die Anerkennung Österreichs als Einwanderungsland zum weitgehend anerkannten Ziel staatlicher Politik. Dasselbe gilt auch für Deutschland, vgl. Albert Scherr/Çiğdem Inan, Leitbilder in der politischen Debatte. Integration, Multikulturalismus und Diversity, in: Frank Gesemann/Roland Roth (Hrsg.), Handbuch Lokale Integrationspolitik, Wiesbaden 2018, S. 201-226, hier S. 202.

6 Ebd., S. 201.

7 Dies sind die Bereiche, die die Tiroler Landesregierung zur Förderung der Integration von Geflüchteten anbietet. Amt der Tiroler Landesregierung, Integration von Flüchtlingen in Tirol, o. D., [https://www.tirol.gv.at/gesellschaftsoziales/integration/angebote-zur-integration-von-fluechtlingen-in-tirol/], eingesehen 18. 1. 2018. 
insofern bedeutend, als davon ausgegangen wird, dass der allgemeine Gender-Gap ${ }^{8}$ in der Bildung und dem Zugang zum Arbeitsmarkt die weitere soziale Integration von Migrant*innen hemmt.

Konkret wird der Frage nachgegangen, inwiefern eine verstärkte zielgruppenspezifische Integration von Migrant*innen mit minderjährigen Kindern notwendig ist und welche sozialintegrative Bedeutung kommunalen Bildungs- und Beratungseinrichtungen dabei zukommt. Es wird angenommen, dass vor allem Migrant*innen mit minderjährigen Kindern von mangelndem Zugang zu Bildungsangeboten und unzureichender Arbeitsmarktintegration betroffen sind, was weitere Dimensionen des Integrationsprozesses negativ beeinflusst und sie zu einer besonderen Risikogruppe für soziale Isolation macht.

Um sich der Forschungsfrage anzunähern, werden zunächst die wichtigsten Integrationstheorien dargestellt. Es folgt ein kurzer Überblick über die Migrationslage in Innsbruck, bevor im empirischen Teil die Familiensituation, Bildung und Arbeit mit jeweiligem Fokus auf eventuelle geschlechtsspezifische Differenzen näher beleuchtet werden. Diese drei Bereiche werden durch die Ergebnisse qualitativer Leitfadeninterviews mit in Innsbruck lebenden Migrant*innen ergänzt und verglichen. Anschließend wird das Potential kommunaler Bildungseinrichtungen für die verstärkte Integration von Migrant*innen mit minderjährigen Kindern untersucht.

In Bezug auf die theoretischen Aspekte zum Thema der Integration sind vor allem die zwei Assimilationsmodelle des amerikanischen Soziologen Milton M. Gordon ${ }^{9}$ und des deutschen Soziologen Hartmut Esser ${ }^{10}$ zu nennen. Um die Migrationslage in Innsbruck untersuchen zu können, werden vor allem die Daten derTiroler Landesregierung ${ }^{11}$ sowie der Statistik Austria ${ }^{12}$ verwendet. Wertvolle und umfassende Beiträge zur kommunalen Integrationspolitik in Deutschland liefern Frank Gesemann und Roland Roth ${ }^{13}$ in dem von ihnen herausgegebenen Werk. Ebenso bedeutsam für die vorliegende Untersuchung ist der europäische Think-Tank von Mikkel Barslund et al., ${ }^{14}$ der den Gender-Gap in der Integration von Migrant*innen in Europa in Bezug auf den Arbeitsmarkt erforscht.

\section{Theoretischer Teil}

\subsection{Theorien zu Assimilation und Integration}

Im öffentlichen und politischen Migrations- und Integrationsdiskurs wird häufig die Forderung nach Anpassung der Migrant*innen an die Kultur, Wertvorstellungen und

$8 \quad$ World Economic Forum, The Global Gender Gap Report 2017, Cologny-Geneva 2017.

9 Milton M. Gordon, Assimilation in American Life. The Role of Race, Religion and National Origin, New York 1964

10 Hartmut Esser, Aspekte der Wanderungssoziologie. Assimilation und Integration von Wanderern, ethnischen Gruppen und Minderheiten. Eine handlungstheoretische Analyse, Darmstadt-Neuwied 1980; Esser, Integration und ethnische Schichtung.

11 Amt der Tiroler Landesregierung, Demografische Daten Tirol 2016, Innsbruck 2017.

12 Statistik Austria, Migration und Integration. Zahlen, Daten, Indikatoren 2017, Wien 2017.

13 Frank Gesemann/Roland Roth (Hrsg.), Handbuch Lokale Integrationspolitik, Wiesbaden 2018.

14 Mikkel Barslund/Anna Di Bartolomeo/Lars Ludolph, Gender Inequality and Integration of Non-EU Migrants in the EU, in: CEPS Policy Insights 6 (2017), S. 1-13. 
Lebensgewohnheiten der ansässigen Bevölkerung diskutiert. Dabei wird oft eine einseitige Angleichung der Einwandernden an die Mehrheitsgesellschaft gefordert. ${ }^{15}$ Dass ein Assimilationsprozess nicht einseitig verläuft, sondern eine Veränderung der Mehrheitsgesellschaft bedingt, gilt als wissenschaftlich belegt. So basieren neuere Assimilationstheorien in der Transnationalisierungsforschung immer noch vielfach auf den klassischen Migrationstheorien aus den 1960er- und 1980er-Jahren, die im Folgenden vorgestellt werden. ${ }^{16}$

Sowohl Gordon als auch Esser gehen in ihrer Theorie von der Annahme aus, dass durch Migration zwei geschlossene Sozialräume getauscht werden und das herkömmliche kulturelle Orientierungswissen durch neues Wissen in der Aufnahmegesellschaft abgelöst wird. Dabei divergieren sie in den verwendeten Begrifflichkeiten. Gordon unterscheidet in seinem Modell zwei Fälle der Assimilation. Der eine setzt voraus, dass die Handlungsmuster der core group ${ }^{17}$ bzw. Mehrheitsgesellschaft von den Migrant*innen übernommen werden, worunter eine kulturelle und strukturelle Anpassung an die Mehrheitsgesellschaft durch institutionelle Eingliederung verstanden wird. Der andere besagt, dass Einwanderungsgesellschaften einen melting pot ${ }^{18}$ darstellen und sich durch Kontakte zwischen den zwei Gruppen auch die Mehrheitsgesellschaft verändert. Gordon weist folglich in seinem Assimilationsmodell darauf hin, dass Assimilation nicht mit der Aufgabe der Herkunftskultur gleichzusetzen ist, sondern Elemente verschiedener Kulturen meist additiv sind und miteinander verschmelzen. ${ }^{19}$

Mit diesem Ansatz harmoniert auch der Assimilationsansatz Hartmut Essers, wobei sich die Kernbereiche seines 1980 formulierten Assimilationsmodells und des im Jahr 2001 entstandenen Integrationsansatzes decken bzw. ergänzen. ${ }^{20}$ So unterscheidet Esser vier Bereiche der Assimilation, der vier Kernbereiche der Sozialintegration zugeordnet werden können. ${ }^{21}$ (I) Unter der Kulturation oder kognitiven Assimilation werden das Wissen und die Kompetenzen verstanden, die wichtig für die Bildungschancen und den Zugang zum Arbeitsmarkt sind. (II) Die Platzierung oder strukturelle Assimilation meint den gleichberechtigten Zugang zu Arbeits- und Wohnungsmarkt und das Bildungssystem sowie den Erwerb bestimmter Rechte wie etwa der Staatsangehörig-

15 Bommes, Die Rolle der Kommunen, S. 102

16 Gordon, Assimilation in American Life; Esser, Aspekte der Wanderungssoziologie.

17 Gordon, Assimilation in American Life, S. 66.

18 Ebd., S. 78

19 Diesen Ansatz übernahmen beispielsweise die amerikanischen Soziologen Alba und Nee. Sie beschäftigten sich in ihrem Modell vordergründig mit Fragen wie gesellschaftlichen Strukturen und institutionellen Arrangements und deren Einfluss auf den Assimilationsprozess. Sie zeigten durch ihr Modell auf, dass Assimilation durch drei unterschiedliche Prozesse erfolgen kann: Boundary Crossing, Boundary Shifting oder Boundary Blurring. Richard Alba/Nictor Nee, Remaking the American Mainstream. Assimilation and Contemporary Immigration, Cambridge u.a. 2003.

20 Esser, Integration und ethnische Schichtung.

21 Esser, Aspekte der Wanderungssoziologie, S. 5-11; Esser, Integration und ethnische Schichtung, S. 8-15. Esser definiert Integration als "Zusammenhalt von Teilen in einem ,systemischen' Ganzen" und unterscheidet in seinem Integrationskonzept zwischen Systemintegration und Sozialintegration. Erstere meint das reibungslose Funktionieren der Gesellschaft als System, wobei die Teile in einem System als voneinander getrennt betrachtet und analysiert werden. Da Migrations- und Einwanderungsgesellschaften aber soziale Systeme darstellen, die sich durch soziale Relationen konstituieren, ist für die vorliegende Untersuchung vor allem die Sozialintegration von Bedeutung. Esser, Integration und ethnische Schichtung, S. 1-5. 
keit. (III) Unter Interaktion oder sozialer Assimilation versteht Esser die sozialen Kontakte mit Einheimischen und Angehörigen anderer Ethnien, Freundschaftsbeziehungen, Nachbarschaftsbeziehungen oder andere Gruppenkontakte. (IV) Die Identifikation oder identifikative Assimilation bezieht sich schließlich auf die Identifikation mit der Aufnahmegesellschaft im Sinne der Entwicklung eines lokalen Zugehörigkeitsgefühls.

Beiden Modellen von Gordon und Esser gemeinsam ist die Annahme, dass das Wissen der Migrant*innen aus ihren Herkunftsländern aufgrund der großen Divergenz zum Einwanderungsland für die strukturelle Assimilation hinderlich ist. So bezeichnet Gordon Kultur als "the social heritage of men", die sich der "core culture of the dominant society ${ }^{\prime 22}$ unterordnen und sich dieser anpassen muss. Esser ist ähnlicher Ansicht, indem er Kultur als einheitliches kognitives Netzwerk versteht, das durch den ausgelösten Homogenisierungsdruck eine Assimilation bedingt. ${ }^{23}$

Für eine gelungene Integration müssen nach Essers Modell alle vier Bereiche der Sozialintegration abgedeckt sein. Dies stellt nun insofern eine Herausforderung dar, als in den Bereichen der Ausbildung und dem Zugang zum Arbeitsmarkt ein geschlechtsspezifischer Unterschied besteht, wodurch weitere Bereiche der sozialen Integration gehemmt werden. Bevor die Gewichtung dieser These genauer analysiert wird, soll zunächst ein einleitender Überblick über Migration und Integration in der österreichischen Kommune Innsbruck gegeben werden.

\subsection{Migration, Integration und Geschlecht in Innsbruck}

Der Anteil an Menschen mit Migrationshintergrund in Tirol stieg in den letzten Jahren mit einigen Schwankungen kontinuierlich an. So lebten im Jahr 2016 insgesamt 147.344 Menschen ausländischer Herkunft in Tirol, was einen Prozentsatz von 19,7 der Gesamtbevölkerung ausmacht. ${ }^{24}$ Betrachtet man die Aufteilung dieses Prozentanteils auf die einzelnen Tiroler Bezirke, stellt Innsbruck-Stadt jenen mit dem größten Anteil an Menschen mit Migrationshintergrund mit 32,2 \% dar, was einem Drittel der Bevölkerung entspricht. ${ }^{25}$

Im Hinblick auf die Analyse der Geschlechterverteilung unter den Migrant*innen in Innsbruck-Stadt wird ersichtlich, dass der dort größte Anteil an Frauen mit Migrationshintergrund (I) zwischen 20 und 34 Jahre alt ${ }^{26}$ ist und (II) hauptsächlich aus Syrien (34,8\%), dem Iran (33,1\%), Marokko (29,7\%), dem Irak (29,1\%) und Nigeria (28,5\%) stammt. ${ }^{27}$ Dies entspricht auch der Zielgruppe, die das Angebot bei Frauen aus allen

22 Gordon, Assimilation in American Life, S. 23.

23 Esser, Aspekte der Wanderungssoziologie, S. 69.

24 Die Komponenten, die für die Erstellung dieser Statistik berücksichtigt wurden, waren (I) Menschen mit nicht österreichischer Staatsangehörigkeit und (II) einem Geburtsland, das nicht Österreich ist. Zusätzlich wurden jene Personen mitberechnet, die (III) zwar die österreichische Staatsbürgerschaft besitzen, jedoch nicht in Österreich geboren wurden. Mit $78 \%$ weit mehr als die Hälfte dieser Personen stellen Migrant*innen der ersten Generation dar. Ihr Geburtsland liegt folglich außerhalb von Österreich. Amt der Tiroler Landesregierung, Demografische Daten Tirol 2016, S. 25

25 Ebd., S. 27

26 Ebd., S. 54

27 Ebd., S. 64. 
Ländern (FAAL) hauptsächlich in Anspruch nimmt, was für die folgende Analyse insofern bedeutend ist, als es auch auf die interviewten Migrant*innen zutrifft.

\section{Empirischer Teil: Zielgruppenspezifische Fallanalyse}

\subsection{Forschungsmethode und Datenbasis}

Für die Recherche der vorliegenden Untersuchung sowie die Entwicklung des Interviewleitfadens wurde unter anderem auf statistische Quellen ${ }^{28}$ zurückgegriffen, um einen quantitativen Überblick über Bildung und Arbeitsmarktsituation von Migrant*innen in Tirol zu erhalten. Der Gegenstand dieser Statistiken deckt sich allerdings nur teilweise mit dem Erkenntnisinteresse. So lassen die Datensätze zwar auf den Stand von Bildung und Erwerbstätigkeit der Individuen schließen, nicht aber auf familiäre geschlechtsspezifische Unterschiede.

Ergänzt und verglichen werden die Daten mit zwei qualitativen semistrukturierten Leitfadeninterviews mit zwei immigrierten Frauen, die an den Angeboten der kommunalen Bildungs- und Beratungseinrichtung Frauen aus allen Ländern (FAAL) ${ }^{29}$ teilgenommen haben. Durch die ehrenamtliche Arbeit der Interviewerin mit den Frauen waren sie zu einem Interview für Forschungszwecke bereit. Der Einfachheit halber werden diese im Folgenden als Gewährspersonen (GP) bezeichnet. Eine kurze Beschreibung der Interviewpartnerinnen soll den Umgang mit den erhobenen Daten erleichtern. So wurde GP1 in Tschetschenien geboren, wo die Dauer ihres Schulbesuches jener der Unterstufe in Österreich glich: Sie besuchte die Schule bis etwa zum 14. Lebensjahr. Nach Kriegsausbruch in Tschetschenien 1995 arbeitete sie für das Rote Kreuz, bevor sie 2004 mit Mann und Kindern nach Österreich floh. Hier lebt sie seit bereits 15 Jahren mit ihren vier Kindern, mittlerweile geschieden von ihrem Mann. GP2 stammt aus dem Irak, wo sie die Volksschule, das Gymnasium und anschließend die Universität in Bagdad besuchte. Nach dem Studium der Betriebswirtschaft arbeiteten sie und ihr Mann als Sunnit*innen für Saddam Hussein, weshalb sie aufgrund des Konfliktes mit der Mehrheit der Schiit*innen gezwungen waren, das Land zu verlassen. Im Jahr 2015 eskalierten die Konflikte zwischen den zwei Gruppen und die Familie erreichte Österreich über viele Umwege. Heute leben sie in Völs. In den Interviews konstituiert sich ihre Rolle im Spannungsfeld zwischen Ethnizität, Geschlecht, Familie, Bildung und Arbeit.

Der Leitfaden orientiert sich an den Kategorien "Allgemeine Informationen“, „Bildung”, "Arbeit", Soziale Interaktion" und „Kommunale Bildungseinrichtungen“, worunter hauptsächlich auf FAAL eingegangen wird. Im Zuge der Kategorienbildung wurde versucht, einen möglichst breiten biographischen Rahmen auf der Basis von den von Esser definierten Kategorien der Sozialintegration zu setzen. So wurde versucht, durch die aus-

28 Amt der Tiroler Landesregierung, Demografische Daten Tirol 2016; Amt der Tiroler Landesregierung, Gleichstellungsbericht Tirol. Frauen und Männer in Tirol, Wien 2016; Statistik Austria, Migration und Integration. Zahlen, Daten, Indikatoren 2017, Wien 2017.

29 Frauen aus allen Ländern. Bildungs- und Beratungseinrichtung, Innsbruck 2014, [https://frauenausallenlaendern. org/impressum/], eingesehen 29. 12. 2017. 
gewählten Kategorien einen Einblick in die Integrationsbereiche Kulturation (Bildung), Platzierung (Arbeit) sowie Interaktion (soziale Interaktion) zu erhalten. Der Bereich der Identifikation wurde durch Fragen zur kommunalen Bildungseinrichtung abgedeckt.

Zur Durchführung der Interviews wurde ein informeller Ort in einem Kaffeehaus gewählt, damit sich die Interviewten nicht zu "Datenlieferanten" ${ }^{\prime 30}$ instrumentalisiert fühlten. Bei den Interviews bzw. Gesprächen selbst wurde von Seiten der Interviewerin

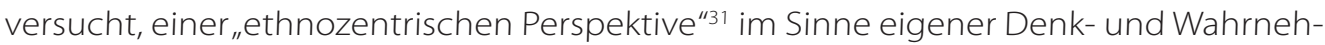
mungsweisen durch größtmögliche persönliche Distanz zu diesen aus dem Weg zu gehen. Gleichzeitig wurde Wert auf das zwischenmenschliche Verhältnis als Ethik der Feldforschung und in diesem Sinne persönliche "Teilnahme"32 an den Erzählungen der Interviewten gelegt.

\subsection{Migration und Gender-Gaps}

Obwohl im Vergleich zum Jahr 2016 zurückgegangen, existieren geschlechtsspezifische Unterschiede vor allem in den Bereichen der ökonomischen und politischen Partizipation, wie die letzte Analyse des World Economic Forum im "Global Gender Gap Report ${ }^{\prime \prime 33}$ offenlegte. Dieser geschlechtsspezifische Unterschied ist auch für die Gruppe der Migrant*innen und deren Integration wirksam.

Der sozialen Gruppe und der gesellschaftlichen Isolation wurden in den letzten Jahren vermehrt Aufmerksamkeit gewidmet. Während in der Schweiz bereits vor mehr als zehn Jahren in unterschiedlichen Studien belegt wurde, dass Migrant*innen mit Erziehungspflichten und ohne Erwerbsarbeit zu den Risikogruppen gehören und am stärksten von Desintegration und sozialer Isolation betroffen sind, rückte das Thema in Österreich erst später in den Fokus der Forschung. Ausschlaggebend war dafür vor allem der oft schlechte gesundheitliche Zustand der Migrant*innen. ${ }^{34}$ Durch diese hauptsächlich aus dem Gesundheitsbereich durchgeführten Studien kristallisiert sich eine Gesellschaftsgruppe heraus, die mitunter zu den am stärksten benachteiligten gezählt werden muss. So betrifft dieses Muster sozialer Isolation vor allem Frauen mit Migrationshintergrund, die in patriarchalen Strukturen aufgewachsen sind, traumatisierende Flucht- und/oder Migrationserfahrungen erlebt haben, als Angehörige einer ethnischen Minderheit rechtlichen und sozialen Diskriminierungen ausgesetzt sowie als Frauen im Beruf benachteiligt sind.

30 Roland Girtler, Methoden der Feldforschung, Wien u. a. 2001³ S. 171.

31 Ebd., S. 123-124.

32 Ebd., S. 116-117.

33 World Economic Forum, The Global Gender Gap Report 2017.

34 Studien aus der Schweiz: Bundesamt für Statistik, Migration und Integration. Ausländerinnen und Ausländer in der Schweiz, Neuchatêl 2004; Bundesamt für Migration, Probleme der Integration von Ausländerinnen und Ausländern in der Schweiz. Bestandesaufnahme der Fakten, Ursachen, Risikogruppen, Maßnahmen und des integrationspolitischen Handlungsbedarfs, Bern 2006; Studien aus Österreich: Internationale Organisation für Migration, Soziale Exklusion und Gesundheit von Migrant*innen in Österreich, Wien 2003; Bundesministerium für Gesundheit und Frauen, Österreichischer Frauengesundheitsbericht 2010/2011, Wien 2010; Liesl Frankl/ Wolfgang Kratky/Michaela Krottendorfer, Aktion Gesunde Seele. Aufsuchendes Programm zur Förderung der psychischen Gesundheit in bildungs- und sozial benachteiligten Migrant*innenfamilien. Endbericht, Wien 2013; Gesundheit Österreich GmbH, Migration und Gesundheit. Literaturbericht zur Situation in Österreich. Im Auftrag der Arbeiterkammer Wien und des Bundesministeriums für Gesundheit, Wien 2015. 
Aus diesem Grund verdient die Untersuchung von migrierten Frauen mit minderjährigen Kindern und deren Arbeitsmarktintegration aus zwei Gründen besondere Aufmerksamkeit. Erstens konzentrieren sich die Lebensbereiche weiblicher Migrant*innen vielfach auf Haushalt und Kindererziehung, während Männer arbeiten. Damit zusammenhängend erhalten Frauen weniger Chancen für den Zugang zu Sprach- und Bildungskursen. Zweitens beeinflusst die Beherrschung der Sprache der Mehrheitsgesellschaft den Zugang zum Arbeitsmarkt, weshalb sich deren Unkenntnis auch auf die Arbeitsmarktintegration negativ auswirkt. In anderen Worten ist der Arbeitsmarkt ohne Kenntnisse der deutschen Sprache nur schwer zugänglich. Die Folge des erschwerten Zugangs zu Bildung und Arbeitsmarkt ist eine doppelte Isolation von der zielspezifischen Gruppe mit den Merkmalen (I) Frau (II) mit Migrationshintergrund und (III) mit minderjährigen Kindern. Die Gewichtung dieser Thesen soll im Folgenden genauer untersucht werden.

\subsection{Familie}

Um die Lebensbedingungen der Zielgruppe von Migrantinnen zu untersuchen, muss im Sinne von Gordon und Esser zunächst der Unterschied zwischen den Herkunftsländern und Österreich als Einwanderungsland thematisiert werden, da die Migrant*innen wesentlich von ersteren geprägt sind.

Zum einen gilt anzumerken, dass westliche Wohlfahrtsgesellschaften wie Österreich oder Deutschland demokratische Partizipation, Gleichberechtigung und individuelle Freiheit bieten, während südliche oder östliche Länder traditionell mit Herrschaft, Gewalt und mehr oder weniger stark ausgeprägten patriarchalen Strukturen verbunden werden. ${ }^{35}$ Soziale Räume, in denen diese Gegensätze in Form binärer Spaltungen sichtbar werden, sind Familien. Durch die Einwanderung von Familien aus stärker patriarchal geprägten Regionen können diese durch die genannten Gegensätze zum Raum des Ausschlusses und der Isolation werden. ${ }^{36}$ Dies wird auch im Interview mit der aus Tschetschenien stammenden GP1 ersichtlich:

„Bei uns in Tschetschenien ist es so, dass man keinen Kontakt mit anderen Männern hat. Auch nicht mit tschetschenischen. Das ist eine andere Mentalität. Ich hatte nur Kontakte mit männlichen Mitgliedern aus meiner Familie. Andere Männer darfst du zwar grüßen und Hallo sagen, aber treffen darfst du dich nur mit Frauen." ${ }^{13}$

35 Stuart Hall, Rassismus als ideologischer Diskurs, in: Nora Räthzel (Hrsg.), Theorien über Rassismus, Hamburg 2000, S. 7-16, hier S. 13; Thomas Geisen/Tobias Studer/Erol Yildiz, Migration und Familie im Kontext von Bildung, Gender und Care. Eine Einleitung, in: Thomas Geisen/Tobias Studer/Erol Yildiz (Hrsg.), Migration, Familie und soziale Lage. Beiträge zu Bildung, Gender und Care, Wiesbaden 2013, S. 1-33, hier S. 3; Stephan Marik-Lebeck/ Josef Kytir, Längsschnittanalyse der Erwerbsverläufe der Migrationskohorten 2010, 2011 und 2012, in: Friedrich Altenburg u. a. (Hrsg.), Migration und Globalisierung in Zeiten des Umbruchs, Krems 2017, S. 237-252.

36 Iman Attia, Die, westliche Kultur' und ihr Anderes. Zur Dekonstruktion von Orientalismus antimuslimischem Rassismus, Bielefeld 2009, S. 33-34; Thomas Geisen/Tobias Studer, Culture matters! Zur Bedeutung von Kultur im Kontext von Migration, in: Bettina Gruber/Daniela Rippitsch (Hrsg.), Migration. Perspektivenwechsel und Bewusstseinswandel als Herausforderung für Stadt und Gesellschaft, Klagenfurt-Celovec 2012, S. 15-33, hier S. 16. Laura Volgger, Interview mit GP1, Innsbruck, 1. 2. 2018, Aufnahme bei der Verfasserin, Z. 281-284. 
Während Männern der Kontakt mit anderen Menschen beider Geschlechter erlaubt ist, dürfen Frauen nur mit anderen Frauen und männlichen Familienmitgliedern kommunizieren, was sich im Falle von ausbleibenden Familienzusammenführungen als schwierig erweist.

Zum anderen hat Migration die Geschlechterverhältnisse in Familien verändert, die sehr unterschiedlich auf die Herausforderungen im Einwanderungsland reagieren. ${ }^{38}$ Diese Herausforderungen bedingen unter anderem Veränderungen, Entwicklungen und Anpassungen von der Elterngeneration, was nicht nur Vater und Mutter selbst, sondern auch deren Beziehung zueinander betrifft. Dadurch werden bisher bekannte Geschlechterrollen hinterfragt, wodurch patriarchale Strukturen herausgefordert werden. ${ }^{39}$ So beschreibt die aus dem Irak stammende GP2, dass sie anfangs Angst hatte, in der Anwesenheit von Männern Deutsch zu sprechen, ${ }^{40}$ während sie nach zwei Jahren viele Freunde beider Geschlechter durch den Besuch des vom Verein Multikulturell organisierten Kontaktcafés in Innsbruck erhielt. ${ }^{41}$ Zudem pflegt sie zu ihrem männlichen Nachbarn ein gutes Verhältnis, den sie als ihren „Bruder"42 bezeichnet. Noch deutlicher wird die Herausforderung an patriarchale Strukturen am Fallbeispiel von GP1. Während diese Frau in den zehn Jahren des Aufenthalts in Österreich "immer zu Hause "43 bei ihren Kindern blieb und der Mann arbeitete, trennte sie sich vor einigen Jahren von ihrem Mann und besuchte einen Deutschkurs. ${ }^{44}$

Diese Emanzipationsprozesse, die sich im Zusammentreffen der Einwanderungsgesellschaft mit der Mehrheitsgesellschaft entwickeln, weisen darauf hin, dass (I) entgegen der Meinung vieler Kritiker*innen ${ }^{45}$ patriarchale Strukturen in Familien von Migrant*innen durchaus anzutreffen sind und zur Benachteiligung der Frauen im Sinne einer sozialen Isolation führen, wie später noch aufgezeigt wird. (II) Gleichzeitig werden patriarchale Strukturen durch das Zusammentreffen herausgefordert und angepasst.

\subsection{Bildung}

Nun soll der Bereich der Bildung von Migrant*innen in Österreich untersucht werden, worunter vor allem der schulische Bildungsverlauf verstanden wird. Der Zusammenhang zwischen Bildung, Geschlecht und Migration wurde bereits häufig untersucht. So ergab die Analyse unabhängiger Studien einen geschlechtsspezifischen Unterschied im Bildungsbereich bei Menschen mit Migrationserfahrung, wobei Männer einen höheren Bil-

38 Ursula Apitzsch/Mechtild M. Jansen (Hrsg.), Migration, Biographie und Geschlechterverhältnisse, Münster 2003; Elisabetta Zontini, Transnational Families, Migration and Gender. Moroccan and Filipino Women in Bologna and Barcelona, New York 2010; Schahrzad Farrokhzad u. a., Verschieden - Gleich - Anders? Geschlechterarrangements im intergenerativen und interkulturellen Vergleich, Wiesbaden 2011; Geisen/Studer, Culture matters!, S. 15-33. Pierrette Hondagneu-Sotelo/Ernestine Avila, "I'm Here, But I'm There". The meanings of Latina transnational motherhood, in: Gender and Society 11 (1997), S. 548-571, hier S. 555; Zontini, Transnational Families, S. 33-35; Geisen/Studer/Yildiz, Migration und Familie im Kontext von Bildung, Gender und Care, S. 5-6.

40 Laura Volgger, Interview mit GP2, Innsbruck, 13. 2. 2018, Aufnahme bei der Verfasserin, Z. 270-272.

41 Innsbrucker Verein Multikulturell, o. D., [www.migration.cc], eingesehen 2. 1. 2018.

42 Volgger, Interview mit GP2, Z. 256.

43 Volgger, Interview mit GP1, Z. 134-135.

44 Ebd., Z. 46.

45 Azizah Y. al-Hibri (Hrsg.), Women and Islam, Richmond 1982; Deniz Kandiyoti, Bargaining with patriarchy, in: Gender and Society 2 (1988), Nr. 3, S. 274-290. 
dungsgrad aufweisen als Frauen. ${ }^{46}$ Betrachtet man etwa den Gleichstellungsbericht der Tiroler Landesregierung von 2016, ${ }^{47}$ zeigt sich, dass das Bildungsniveau von Migrant*innen in Tirol zwar heterogen ist, der Anteil der Frauen mit Migrationshintergrund mit maximal Pflichtschulabschluss aber deutlich über dem der Männer liegt. Während Frauen aus dem ehemaligen Jugoslawien zu 70 \% eine schulische Ausbildung aufweisen, die maximal den Pflichtschulabschluss erreicht, trifft dies bei männlichen Migranten aus Ex-Jugoslawien nur zu 52 \% zu. Frauen aus der Türkei haben zu 89 \% keinen über die Pflichtschule hinausgehenden Abschluss erlangt, Männer zu $72 \%$. Bei Türkinnen weist somit nur jede Zehnte eine über den Pflichtschulabschluss hinausgehende Ausbildung auf.

In Bezug auf die Kategorien Bildung und Familie ist die Datenlage zu Frauen bzw. Müttern mit Migrationshintergrund immer noch unzureichend. Während im Zuge der vermehrten Einwanderungen türkischer Migrant*innen in deutschsprachige Länder vor allem die Lebensbereiche türkischer Schüler*innen untersucht wurden, ${ }^{48}$ bleibt die Erforschung von Müttern mit minderjährigen Kindern weitgehend unbeleuchtet. Der Zusammenhang zwischen Bildung, Geschlecht und Familie im Kontext von Migration wurde in den geführten Interviews deutlich. So führte etwa GP1 die nach 14-jährigem Aufenthalt in Österreich immer noch unzureichenden Deutschkenntnisse auf patriarchale Familienstrukturen zurück: „Mein Mann hat sehr gut Deutsch gesprochen. Ich nicht. Ich bin immer zu Hause. Erst vor zwei oder drei Jahren bin ich zum ersten Mal in einen Deutschkurs gegangen."49 Der Grund für die Konzentration auf den häuslichen und familiären Raum liegt in der Kindererziehung:

„Ungefähr 14 Jahre lang habe ich keinen Deutschkurs besucht wegen meinen Kindern. Meine Kinder waren sehr klein und das erste ist 2003 geboren. Das nächste dann 2008. Wenn sie so klein sind, brauchen Kinder viel Zeit. Deswegen hatte ich leider für einen Deutschkurs keine Zeit. Sobald die Kinder größer wurden und das kleinste Kind auch in die Schule ging, besuchte ich den Deutschkurs." ${ }^{\prime 50}$

Dass sich dieser klar definierte Rollenstereotyp der Mutter nicht nur bei Müttern mit minderjährigen Kindern, sondern auch bei jenen mit volljährigen Kindern negativ auf den Zugang zu Sprachkursen auswirkt, thematisiert auch die GP2:

46 Österreichischer Integrationsfonds, Aktuelles zu Migration und Integration. Migrant*innen in Österreich (Fact Sheet Nr. 28), Wien 2018; Amt der Tiroler Landesregierung, Gleichstellungsbericht; Statistik Austria, Migration und Integration.

47 Amt der Tiroler Landesregierung, Gleichstellungsbericht, S. 28.

48 Franz Hamburger/Tarek Badawia/Merle Hummrich (Hrsg.), Migration und Bildung. Über das Verhältnis von Anerkennung und Zumutung in der Einwanderungsgesellschaft (Schule und Gesellschaft 35), Wiesbaden 2005; Albert Tanner u. a. (Hrsg.), Heterogenität und Integration. Umgang mit Ungleichheit und Differenz in Schule und Kindergarten, Zürich 2006; Nevâl Gültekin, Bildung, Autonomie, Tradition und Migration. Doppelperspektivität biographischer Prozesse junger Frauen aus der Türkei, Wiesbaden 2013.

50 Volgger, Interview mit GP1, Z. 152-156. 
"Ich kann Arabisch sprechen und schreiben. Aber zu Hause ist oft nicht viel Zeit, um Deutsch zu lernen. Mein Sohn ist 22 Jahre alt und geht nach dem Deutschkurs zur Universität. Dann muss ich für alle kochen." ${ }^{451}$

Zusammenfassend lässt sich in Bezug auf die Integration von Migrant*innen mit minderjährigen Kindern in den Bildungsbereich festhalten, dass Mütter aufgrund der geschlechtsspezifischen Rollenverteilung und der Konzentration auf Haushalt und Kindererziehung einen erschwerten Zugang zu Sprach- und Bildungskursen haben, was die Kulturation oder kognitive Assimilation nach Esser aufgrund des dadurch entstehenden Zeitmangels behindert. Inwiefern die im Bildungsbereich nachgewiesene strukturelle Vernachlässigung dieser spezifischen Zielgruppe auch in der Arbeitsmarktintegration existiert, soll im Folgenden untersucht werden.

\subsection{Arbeitsmarktintegration}

Erst kürzlich erschienen mehrere nennenswerte Studien, die sich mit der Bildung von Migrant*innen und deren Bedeutung für die Arbeitsmarktintegration auseinandersetzen. ${ }^{52}$ Während die unterschiedlichen sozioökonomischen Bedingungen der Migrant*innen in diesen Studien vielfach berücksichtigt werden, ist dies in der praktischen Arbeitsmarktintegration selten der Fall. ${ }^{53}$ Hier wird der Zusammenhang zwischen Migration, Geschlecht, Familie, Beruf und Arbeit deutlich, wobei sich ein geschlechtsspezifischer Unterschied zeigt. ${ }^{54}$ Nach Angaben der Österreichischen Kommission für Migrations- und Integrationsforschung bestand 2017 bei der Arbeitslosenquote nur ein marginaler Unterschied zwischen Männern und Frauen mit Migrationserfahrung, während die Diskrepanz in der Erwerbstätigkeit merklich höher war. ${ }^{55}$ So waren in diesem Jahr 69 \% der Migranten und lediglich 58 \% der Migrantinnen erwerbstätig. ${ }^{56}$

Ein besonders großer gap in der Arbeitsmarktintegration besteht zwischen Frauen mit und ohne Migrationshintergrund mit minderjährigen Kindern. Während jene mit Migrationshintergrund und Kindern unter 18 zu $39 \%$ nicht und zu 38 \% teilzeitig erwerbstätig waren, ergibt sich bei Frauen ohne Migrationshintergrund eine Verteilung

51 Volgger, Interview mit GP2, Z. 128-130.

52 Eurofound, Approaches to the labour market integration of refugees and asylum seekers, Publications Office of the European Union, Luxembourg 2016; European Commission, Challenges in the labour market integration of asylum seekers and refugees, EEPO ad hoc request, Publications Office of the European Union, Luxemburg 2016; European Commission, Employment and Social Developments in Europe. Annual Review 2016, Luxemburg 2016; Holger Bonin, The Potential Economic Benefits of Education of Migrants in the EU (EENEE Analytical Report 31), Prepared for the European Commission, Brüssel 2017; Uuriintuya Batsaikhan/Zsolt Darvas/Inês Gonçalves Raposo, People on the move. Migration and mobility in the European Union. Europe's immigration and integration challenges: Financial and labour market dimensions, Rome 2018.

53 Barslund/Di Bartolomeo/Ludolph, Gender Inequality and Integration, S. 3.

54 Dass Frauen häufig weniger entlohnt werden als Männer, obwohl sie einen höheren Ausbildungsgrad besitzen, belegt der aktuell erschienene Global Gender Gap Report 2017. Von insgesamt 144 untersuchten Ländern befindet sich Österreich in Bezug auf die wirtschaftliche Partizipation und die entsprechende Entlohnung auf Platz 80. World Economic Forum, The Global Gender Gap Report, S. 70. Im Folgenden wird unter Arbeitsmarktintegration jedoch nicht die Entlohnung, sondern lediglich der Zugang zum Arbeitsmarkt von Migrant*innen verstanden. Während Migranten zu 13,4\% arbeitslos waren, beträgt der Prozentsatz bei Migrant*innen 13,7 \%. Statistik Austria, Migration und Integration, S. 63 
von $15 \%$ zu $60 \% .^{57}$ Inwiefern die Bevorzugung einer Nicht-Erwerbstätigkeit und der mangelnde Wille zur Erwerbstätigkeit hierbei eine Rolle spielen, ist schwer feststellbar. Dass strukturelle Diskriminierung besonders in kleineren Kommunen trotzdem vorhanden ist und dadurch der Integrationsprozess dieser Zielgruppe beeinträchtigt wird, verdeutlicht das Fallbeispiel von GP1:

„Ich suche eine Arbeit, aber es ist schwierig. Sehr schwierig. Ich hätte zwar eine Arbeit gefunden, doch sobald der Arbeitgeber den Tschador gesehen hat, sagte er, dass er doch nicht will, da die Leute Angst haben." ${ }^{58}$

Deutlicher wird die strukturelle Benachteiligung weiblicher Migranten bei überqualifizierter Beschäftigung. $27 \%$ der Frauen mit Migrationshintergrund übten 2017 einen Beruf aus, für den sie überqualifiziert waren, bei Männern waren es lediglich $18 \% .{ }^{59}$ Während Frauen mit Migrationserfahrung am häufigsten in der Branche der Unternehmensdienstleistungen wie der Gebäudereinigung oder Kraftwagenvermietung tätig waren, arbeiteten Männer zum Großteil in der Gastronomie. ${ }^{60}$ Dies ist insofern problematisch, als im Unternehmensdienstleistungssektor im Unterschied zur Gastronomie nur begrenzte Kontaktmöglichkeiten zu anderen Personen bestehen, was sich auch auf die Sprachkompetenz auswirkt. Dies wird etwa am Fallbeispiel von GP2 ersichtlich:

„Aber ich brauche Arbeit, eine Arbeit, bei der ich Deutsch sprechen kann. Ich brauche eine Arbeit, bei der ich Kontakte knüpfen kann, auch wenn ich nur ein oder zwei Worte am Tag sprechen kann. Das ist egal. Aber in meiner anderen Arbeit [Anm.: hat in einer Schule geputzt und aufgeräumt] habe ich nie gesprochen und ich war immer alleine. Ich brauche eine Arbeit, wo ich sprechen kann." ${ }^{\prime 61}$

Der Einfluss der Sprache für den Zugang zum Arbeitsmarkt und gleichzeitig die Bedeutung der Erwerbstätigkeit für das Erlernen der Sprache wurden wissenschaftlich breit diskutiert. So zeigte etwa Esser in seinen empirischen Studien, dass Defizite in der Beherrschung der jeweiligen Landessprache für Diskriminierungen und soziale Distanzen eine große Rolle spielen. ${ }^{62}$

57 Statistik Austria, Migration und Integration, S. 55.

58 Volgger, Interview mit GP1, Z. 309-311. Anm.: Die Frau verhüllt mit ihrem Tschador gemäß dem österreichischen Anti-Gesichtsverhüllungsgesetz vom 1. 10. 2017 (Österreichisches Bundeskanzleramt, Bundesrecht konsolidiert. Gesamte Rechtsvorschrift für Anti-Gesichtsverhüllungsgesetz, erlassen am 1. 10. 2017, Wien 2017) lediglich Kopf und Körper, nicht aber das Gesicht, wobei ihr trotzdem die Arbeitsmarktintegration aufgrund des Tschadors verwehrt wurde.

59 Statistik Austria, Migration und Integration, S. 61.

60 Ebd., S. 58.

61 Volgger, Interview mit GP2, Z. 134-137.

62 Europarat, Integration tests: helping or hindering integration? Report. Provisional version, Brüssel 2013; Pew Research Center, Spring 2016 Global Attitudes Survey. July 2016, o. O. 2016; Hartmut Esser, Sprache und Integration. Konzeptionelle Grundlagen und empirische Zusammenhänge. Arbeitspapier 7, Wien 2006; Hartmut Esser, Migration, Sprache und Integration. AKI-Forschungsbilanz 4, Berlin 2006; Piet van Avermaet, Tests for citizenship and integration in Europe. A critical reflection, in: Barbara Haider (Hrsg.), Deutsch über alles? Sprachförderung für Erwachsene, Wien 2011, S. 59-76. 
Durch den erschwerten Zugang zu Bildungs- und Sprachkursen sowie der strukturelIen Benachteiligung in der Arbeitsmarktintegration ${ }^{63}$ stellen Frauen mit Migrationserfahrung und minderjährigen Kindern eine besondere Risikogruppe für soziale Isolation dar. Dies birgt zwei wesentliche Gefahren. Die eine besteht im Festfahren von Einwanderungsminderheiten in den untersten Gesellschaftsbereichen. Da es aufgrund des vom deutschen Soziologen Petrus Han beschriebenen withdrawal keine sub-stantielle Beziehung zwischen Einwanderungsminderheit und Mehrheitsgesellschaft mehr gibt, lebt die Minderheit isoliert in ihrer ethnischen Kultur. Die Segregation liegt dann vor, wenn diese Isolation der sozialen Minderheit auf die bewusste Ausgrenzung durch die Mehrheitsgesellschaft zurückgeht. ${ }^{64}$ In diesem Sinne sind auch strukturelle Ausgrenzungen am Arbeitsmarkt als Segregation zu verstehen. Dies führt dazu, dass Einwandererminderheiten durch die räumliche Isolation und den Ausschluss aus den Arbeitsund Bildungsstrukturen im untersten Gesellschaftsbereich behaftet bleiben, was vor allem für Migrant*innen mit minderjährigen Kindern gilt. ${ }^{65}$

Die andere damit zusammenhängende Gefahr bezieht sich nicht auf die Stellung der Migrant*innen im gesellschaftlichen Kollektiv, sondern auf jene innerhalb der eigenen Familie. So kann durch die mangelnde Sozialintegration eine Isolation innerhalb des familiären Raumes erfolgen. Dies wird etwa am Fallbeispiel von GP1 ersichtlich:

„Wenn ich mit ihnen [meinen Kindern] Tschetschenisch rede, verstehen sie mich oft nicht und sagen, ich solle mit ihnen bitte Deutsch reden. Wenn sie untereinander sind, reden sie Deutsch, nicht Tschetschenisch. Auch, wenn sie z.B. spielen oder Hausübungen machen, reden sie Deutsch. Immer Deutsch. Ich sage dann oft, dass sie bitte Tschetschenisch reden sollen, da ich sie nicht verstehe. Dann lachen meine vier Kinder, da Mama kein Deutsch versteht." ${ }^{\prime 6}$

Die Konfrontation mit der deutschen Sprache im Umgang mit anderen Familienmitgliedern stellt somit eine Herausforderung für Frauen dar, die der Sprache nicht mächtig sind. Diesbezüglich wird die Schwierigkeit der unterschiedlich schnell verlaufenden Integration von Eltern und Kindern deutlich. Dass dieser Unterschied einen ernstzunehmenden Problembereich darstellt, betonte 2018 der schwedische Regionalpräsident Berntsson: „The children go to school and are integrated quickly. But parents take too much time, and this creates differences between the children and the grownups. ${ }^{\prime 67}$ Der Fakt, dass die Kinder von GP1 in Österreich geboren wurden und Kindergarten und Schule dort besuchten, führt nicht nur dazu, dass die Kinder im Gegensatz zu den Eltern und dabei vor allem der Mütter, die nicht erwerbstätig sind, die deutsche

63 Barslund/Di Bartolomeo/Ludolph, Gender Inequality and Integration, S. 1-13.

64 Petrus Han, Soziologie der Migration. Erklärungsmodelle, Fakten, politische Konsequenzen, Perspektiven, München $2016^{4}$, S. 22.

65 Milton Gordon, Assimilation in America. Theory and Reality, in: Nerman R. Yetman/Henry Steele (Hrsg.), Majority and Minority. The dynamics of race and ethnicity in American life, Boston 1975, S. 272 - 284, hier S. 278.

66 Volgger, Interview mit GP1, Z. 120-125.

67 Magnus Berntsson in der Diskussion zu "Urban responses to refugees" am 25. Jänner 2018, in: Friends of Europe, Solutions to refugee integration lie at the local level. 
Sprache schneller lernen, sondern gleichzeitig, dass sie die L ${ }^{68}$ der Eltern nicht oder unzureichend sprechen. Dadurch wird das Schaffen einer gemeinsamen Kommunikationsbasis erschwert. Durch die resultierende Isolation in der eigenen Familie, die mitunter eine starke emotionale Belastung darstellen kann, muss somit von einer durch unzureichende Integration resultierenden doppelten Isolation gesprochen werden. ${ }^{69}$

\subsection{Sozialintegrative Leistung der Kommunen}

Eine Möglichkeit, um soziale Isolation zu verhindern und Migrant*innen mit minderjährigen Kindern gesellschaftlich zu integrieren, stellen kommunale Bildungs- und Beratungseinrichtungen dar. Da Kommunen, Städte und Gemeinden erst in den letzten Jahren vermehrte Aufmerksamkeit in europäischen und nationalen Debatten über die Integrations- und Migrationspolitik erhielten, entwickelte sich dieses Politikfeld nur zögerlich und es fehlt an einer systematischen wissenschaftlichen Erforschung von Kommunen im nationalen und europäischen Vergleich. ${ }^{70}$ Dabei haben die Kommunen "significant impacts on the local migrants population"71 in rechtlicher, politisch-partizipatorischer und sozioökonomischer Hinsicht. Aufgrund mangelnder systematischer Daten im Hinblick auf kommunale Bildungseinrichtungen soll im Folgenden das Konzept der Innsbrucker Bildungs- und Beratungseinrichtung Frauen aus allen Ländern $(F A A L)^{72}$ exemplarisch untersucht werden. Gegründet 2001 für Frauen mit Migrationshintergrund oder Fluchterfahrung, bietet die Einrichtung mehrere Angebote mit dem Ziel, die Integration der Frauen zu verbessern und gleichzeitig Antirassismusarbeit zu leisten.

Zunächst ist die Bedeutung kommunaler Bildungseinrichtungen für das Erlernen mündlicher und schriftlicher Fähigkeiten zu nennen. Durch Sprach-, Alphabetisierungs- und Basisbildungskurse wird die Kulturation oder kulturelle Integration der Migrant*innen gefördert, wobei der gleichzeitigen Kinderbetreuung wie aufgezeigt eine besondere Bedeutung zukommt. Diese kulturelle Integration stellt nach Esser den „Schlüssel für jede nachhaltige Sozialintegration"73 dar. So verdeutlicht etwa GP1, dass ein enger Zusammenhang zwischen mangelnder Sprachkompetenz und sozialer Isolation besteht:

68 Anm.: L1 ist die erste Sprache einer Person, oft als "Muttersprache“ bezeichnet.

69 Die Gewichtung dieser These kann gewiss nur durch eine breite Untersuchung gestützt werden. Diese erweist sich aufgrund der gesellschaftlichen Isolation und der folglich erschwerte Zugänglichkeit zu diesen Frauen allerdings als schwierig. Demgemäß liefert die für die vorliegende Arbeit verwendete Datenbasis einen zwar kleinen Beitrag, auf den jedoch durch weitere empirische Untersuchungen aufgebaut werden kann.

70 Jutta Aumüller, Die kommunale Integration von Flüchtlingen, in: Frank Gesemann/Roland Roth (Hrsg.), Handbuch Lokale Integrationspolitik, Wiesbaden 2018, S. 173-198; Myrte S. Hoekstra/Josef Kohlbacher/Daniel Rauhut, Migration Governance in Three European Cities: New Local Paradigms?, in: Thomas Lacroix/Amandine Desille (Hrsg.), International Migrations and Local Governance. A Global Perspective, Cham 2018, S. 17-38.

71 Michael Alexander, Comparing Local Policies towards Migrants. An Analytical Framework, a Typology and Preliminary Survey Results, in: Rinus Penninx u. a. (Hrsg.), Citizenship in European Cities. Immigrants, local politics and integration policies, New York u. a. 2016, S. 83-99, hier S. 83-84.

72 Frauen aus allen Ländern. Bildungs- und Beratungseinrichtung.

73 Esser, Integration und ethnische Schichtung, S. 10. 
„Bei mir ist es immer so: Wenn man in ein anderes Land zieht, ist man am Anfang immer nur zu Hause, besonders dann, wenn man die Sprache nicht spricht. Wenn man die Sprache nicht spricht, ist das für mich ein großer Nachteil. Dann will ich nur zu Hause sein. Wenn man gut Deutsch spricht, dann geht man gerne hinaus." ${ }^{174}$

Zudem schaffen kommunale Bildungseinrichtungen interethnische Begegnungsräume. Dies ist einerseits insofern hilfreich, als die Migrant*innen die social norms und etiquette der Mehrheitsgesellschaft kennenlernen, deren Missachtung nach Alba und $\mathrm{Nee}^{75}$ am signifikantesten für die Aufrechterhaltung sozialer Diskriminierung ethnischer Minderheitengruppen sind. Andererseits helfen die Kontakte mit anderen Migrant*innen dabei, negative Selbstzuschreibungen zu revidieren. Dies wird im Interview mit GP1 ersichtlich, deren Selbstbild stark defizitär behaftet war: „Ich hatte auch mit den anderen Frauen viel Kontakt und dachte mir irgendwann, vielleicht bin ich auch gut und nicht

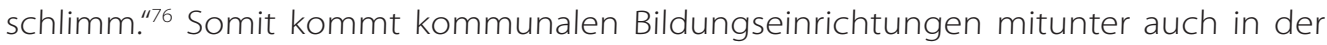
Förderung der Interaktion oder sozialen Integration eine wesentliche Bedeutung zu.

Solche Einrichtungen erhalten eine zusätzliche Gewichtung für die Platzierung oder strukturelle Integration in die Mehrheitsgesellschaft, wenn auch mehr indirekt. So beraten sie etwa über den Zugang zum Arbeits- und Wohnungsmarkt, über Gleichberechtigungsfragen oder helfen beim Ausfüllen von Formularen. ${ }^{77}$

Durch den meist überschaubaren Rahmen der Kursgröße wird das Schaffen einer Vertrauensbasis zwischen Trainer*innen und Migrant*innen geschaffen, wobei kommunale Bildungseinrichtungen meist ungezwungene Räume des sozialen Austausches bieten. So nennt etwa GP2 den direkten Zusammenhang zwischen sozialer und identifikativer Integration: „Ich finde die meisten Freunde durch das Kontaktcafé und den Deutschkurs. In Völs habe ich besonders viele Freunde. Völs ist mein Land, meine Heimat..78 In diesem Sinne tragen kommunale Bildungseinrichtungen auch zur Identifikation der Migrant*innen mit der Aufnahmegesellschaft nach Esser bei. ${ }^{79}$

\section{Resümee}

Zu Beginn der Arbeit stellte sich die Frage, inwiefern eine zielgruppenspezifische Integration von Migrant*innen mit minderjährigen Kindern notwendig ist, und was kommunale Bildungseinrichtungen hierbei leisten können.

Allgemein ist festzuhalten, dass in den untersuchten Bereichen der Familie, Bildung und des Berufs eine geschlechtsspezifische Differenzierung bei Menschen mit Migrationshintergrund besteht, die jeweils eine Begünstigung sozialer Isolation bei der untersuchten Zielgruppe bedingen.

\footnotetext{
74 Volgger, Interview mit GP1, Z. 138-141.

75 Alba/Nee, Remaking the American Mainstream, S. 133.

76 Volgger, Interview mit GP1, Z. 289-290.

77 Bommes, Die Rolle der Kommunen, S. 101-102.

78 Volgger, Interview mit GP2, Z. 165-166.

79 Esser, Integration und ethnische Schichtung, S. 83.
} 
So ergab die Analyse des familiären Bereichs einerseits, dass sich die Lebensbereiche weiblicher Migrant*innen mit minderjährigen Kindern verstärkt auf die Bereiche Haushalt und Kindererziehung konzentrieren, während Männer mehrheitlich arbeiten. Dies ist unter anderem auf die vielfach patriarchalen Familienstrukturen zurückzuführen, die gleichzeitig durch das Zusammentreffen mit anderen Familienformen in der Mehrheitsgesellschaft moderiert und verändert werden.

Im Bildungsbereich wurde die teils geringe Schulbildung weiblicher Migrant*innen nachgewiesen. Dieser Gegensatz zu männlichen Einwanderern erhält sich durch die verstärkte Konzentration auf den häuslichen Raum und dem folgenden unzureichenden Spracherwerb aufrecht. Dieser beeinflusst wiederum den Zugang zum Arbeitsmarkt.

Im Arbeitsmarkt wird die Auswirkung der genannten Bereiche besonders deutlich. So sind weibliche Migrant*innen mit Kindern unter 18 Jahren seltener erwerbstätig, wodurch weniger Sozialkontakte entstehen und der Erwerb der Sprache der Mehrheitsgesellschaft nicht gefördert wird. Gleichzeitig sind erwerbstätige Migrant*innen vor allem im Unternehmensdienstleistungssektor tätig, wodurch Sozialkontakte ebenfalls nur begrenzt möglich sind und das Erlernen der Fremdsprache am Arbeitsplatz ausbleibt. Folglich erweist sich die Integrationsdimension der Platzierung als schwer erreichbar für die Zielgruppe der Migrant*innen mit minderjährigen Kindern. Dies erschwert gleichzeitig die Kulturation und hemmt den Integrationsbereich der Interaktion. Über die Interaktion vollzieht sich aber "die,Platzierung' der Akteure in den alltäglichen, nicht formellen und nicht in den Märkten verankerten Bereiche der Gesellschaft", 80 was bedeutet, dass mangelnde Integration des einen Bereichs eine Hemmnis der Integration des anderen bedingt.

Wird die Integration in einem der Bereiche gestört oder durch äußere Einflüsse wie strukturelle Zugangsschwierigkeiten zum Arbeitsmarkt gehemmt, was bei der untersuchten Zielgruppe häufig der Fall ist, wird der gesamte Integrationsprozess negativ beeinflusst. Dies führt zu einer doppelten Isolation sowohl in der Gesamtgesellschaft als auch der Familie.

Einen wesentlichen Beitrag zur Integration von Frauen mit Migrationshintergrund und minderjährigen Kindern leisten kommunale Bildungs- und Beratungseinrichtungen. Dabei erweist sich vor allem die Förderung der kulturellen und sozialen Integration als für die weitere Sozialintegration nützlich.

Im Zuge der Beschäftigung mit der Thematik ergaben sich zahlreiche weitere Fragen im Zusammenhang mit Migration, Integration und Isolation. So könnten sich weitere Forschungen etwa mit einer breiteren empirischen Untersuchung der Familien- und Wohnsituation von Migrantinnen und den Auswirkungen auf den Integrationsprozess oder weiteren Fallanalysen der Auswirkungen mangelnder Sozialintegration auf die Familiensituation befassen. Interessant wäre auch ein nationaler Vergleich kommuna- 
ler Bildungseinrichtungen, um die Schaffung internationaler Standards in der lokalen Integration zu unterstützen.

\section{Literaturverzeichnis}

Alba, Richard/Nee, Victor, Remaking the American Mainstream. Assimilation and Contemporary Immigration, Cambridge u. a. 2003.

Alexander, Michael, Comparing Local Policies towards Migrants. An Analytical Framework, a Typology and Preliminary Survey Results, in: Rinus Penninx u. a. (Hrsg.), Citizenship in European Cities. Immigrants, local politics and integration policies, New York u. a. 2016, S. 83-99.

Al-Hibri, Azizah Y. (Hrsg.), Women and Islam, Richmond 1982.

Angenendt, Steffen/Koch, Anne, Global Migration Governance im Zeitalter gemischter Wanderungen. Folgerungen für eine entwicklungsorientierte Migrationspolitik, in: SWP-Studien 8 (2017), S. 1-34, [https://www.swp-berlin.org/fileadmin/contents/products/studien/2017S08_adt_koh.pdf], eingesehen 27.1.2018.

Apitzsch, Ursula/Jansen, Mechtild M. (Hrsg.), Migration, Biographie und Geschlechterverhältnisse, Münster 2003

Attia, Iman, Die,westliche Kultur' und ihr Anderes. Zur Dekonstruktion von Orientalismus und antimuslimischem Rassismus, Bielefeld 2009.

Aumüller, Jutta, Die kommunale Integration von Flüchtlingen, in: Frank Gesemann/Roland Roth (Hrsg.), Handbuch Lokale Integrationspolitik, Wiesbaden 2018, S. 173-198.

Barslund, Mikkel/Di Bartolomeo, Anna/Ludolph, Lars, Gender Inequality and Integration of Non-EU Migrants in the EU, in: CEPS Policy Insights 6 (2017), S. 1-13, [https://www. ceps.eu/system/files/No\%202017-06\%20MB\%20et\%20al\%20Gender\%20Inequality\%20and\%20Integration\%20of\%20Non-EU\%20Migrants\%20in\%20the\%20EU.pdf], eingesehen 25. 1. 2018.

Batsaikhan, Uuriintuya/Darvas, Zsolt/Gonçalves Raposo, Inês, People on the move. Migration and mobility in the European Union. Europe's immigration and integration challenges: Financial and labour market dimensions, Rome 2018, [http://www.iai.it/sites/default/files/migration_180202-zsolt.pdf], eingesehen 2. 2. 2018.

Berntsson, Magnus in der Diskussion zu "Urban responses to refugees" am 25. Jänner 2018, in: Friends of Europe, Solutions to refugee integration lie at the local level, [http:// www.friendsofeurope.org/node/67343], eingesehen 2. 2. 2018.

Bommes, Michael, Die Rolle der Kommunen in der bundesdeutschen Migrations- und Integrationspolitik, in: Frank Gesemann/Roland Roth (Hrsg.), Handbuch Lokale Integrationspolitik, Wiesbaden 2018, S. 99-123.

Bossong, Raphael, Zwänge und Spielräume für eine europäische Grenz- und Flüchtlingspolitik. Kurzfristige Szenarien, in: Stiftung Wissenschaft und Politik 38 (2017), 
S. 1-4, [https://www.swp-berlin.org/fileadmin/contents/products/aktuell/2017A38_ bsg.pdf], eingesehen 12.1. 2018.

Esser, Hartmut, Aspekte der Wanderungssoziologie. Assimilation und Integration von Wanderern, ethnischen Gruppen und Minderheiten. Eine handlungstheoretische Analyse, Darmstadt-Neuwied 1980.

Ders., Integration und ethnische Schichtung (Mannheimer Zentrum für Europäische Sozialforschung, Arbeitspapiere 40), Mannheim 2001.

Ders., Migration, Sprache und Integration. AKI-Forschungsbilanz 4, Berlin 2006.

Ders., Sprache und Integration. Konzeptionelle Grundlagen und empirische Zusammenhänge. Arbeitspapier 7, Wien 2006.

Farrokhzad, Schahrzad u. a., Verschieden - Gleich - Anders? Geschlechterarrangements im intergenerativen und interkulturellen Vergleich, Wiesbaden 2011.

Frankl, Liesl/Kratky, Wolfgang/Krottendorfer, Michaela, Aktion Gesunde Seele. Aufsuchendes Programm zur Förderung der psychischen Gesundheit in bildungs- und sozial benachteiligten MigrantInnenfamilien. Endbericht, Wien 2013, [http://old.fgoe.org// projektfoerderung/gefoerderte-projekte/FgoeProject_3150/69604.pdf], eingesehen 2. 1.2017.

Geisen, Thomas/Studer, Tobias, Culture matters! Zur Bedeutung von Kultur im Kontext von Migration, in: Bettina Gruber/Daniela Rippitsch (Hrsg.), Migration. Perspektivenwechsel und Bewusstseinswandel als Herausforderung für Stadt und Gesellschaft, Klagenfurt-Celovec 2012, S. 15-33.

Geisen, Thomas/Studer, Tobias/Yildiz, Erol, Migration und Familie im Kontext von Bildung, Gender und Care. Eine Einleitung, in: Thomas Geisen/Tobias Studer/Erol Yildiz (Hrsg.), Migration, Familie und soziale Lage. Beiträge zu Bildung, Gender und Care, Wiesbaden 2013, S. 1-33.

Girtler, Roland, Methoden der Feldforschung, Wien u. a. $2001^{3}$.

Gordon, Milton, Assimilation in American Life. The Role of Race, Religion and National Origin, New York 1964.

Ders., Assimilation in America. Theory and Reality, in: Nerman R. Yetman/Henry Steele (Hrsg.), Majority and Minority. The dynamics of race and ethnicity in American life, Boston 1975, S. $272-284$.

Gültekin, Nevâl, Bildung, Autonomie, Tradition und Migration. Doppelperspektivität biographischer Prozesse junger Frauen aus der Türkei, Wiesbaden 2013.

Hall, Stuart, Rassismus als ideologischer Diskurs, in: Nora Räthzel (Hrsg.), Theorien über Rassismus, Hamburg 2000, S. 7-16.

Hamburger, Franz/Badawia, Tarek/Hummrich, Merle (Hrsg.), Migration und Bildung. Über das Verhältnis von Anerkennung und Zumutung in der Einwanderungsgesellschaft (Schule und Gesellschaft 35), Wiesbaden 2005. 
Han, Petrus, Soziologie der Migration. Erklärungsmodelle, Fakten, politische Konsequenzen, Perspektiven, München $2016^{4}$.

Hoekstra, Myrte S./Kohlbacher, Josef/Rauhut, Daniel, Migration Governance in Three European Cities: New Local Paradigms?, in: Thomas Lacroix/Amandine Desille (Hrsg.), International Migrations and Local Governance. A Global Perspective, Cham 2018, S. 17-38.

Hondagneu-Sotelo, Pierrette/Avila, Ernestine, "I'm Here, But I'm There". The meanings of Latina transnational motherhood, in: Gender and Society 11 (1997), S. 548-571.

Kandiyoti, Deniz, Bargaining with patriarchy, in: Gender and Society 2 (1988), S. 274-290.

Marik-Lebeck, Stephan/Kytir, Josef, Längsschnittanalyse der Erwerbsverläufe der Migrationskohorten 2010, 2011 und 2012, in: Friedrich Altenburg u. a. (Hrsg.), Migration und Globalisierung in Zeiten des Umbruchs, Krems 2017, S. 237-252.

Nagy, Boldizsár, Sharing the Responsibility or Shifting the Focus? The Responses of the EU and the Visegrad Countries to the Post-2015 Arrival of Migrants and Refugees, in: Global Turkey in Europe, Working Paper 17 (2017), S. 1-20, [http://www.iai.it/sites/default/files/gte_wp_17.pdf], eingesehen 2. 2. 2018.

Österreichisches Bundeskanzleramt, Bundesrecht konsolidiert. Gesamte Rechtsvorschrift für Anti-Gesichtsverhüllungsgesetz, erlassen am 1. 10. 2017, Wien 2017, [https:// www.ris.bka.gv.at/GeltendeFassung.wxe?Abfrage=Bundesnormen\&Gesetzesnummer $=20009892 \&$ ShowPrintPreview=True], eingesehen 2. 1. 2018 .

Scherr, Albert/Inan, Çiğdem, Leitbilder in der politischen Debatte. Integration, Multikulturalismus und Diversity, in: Frank Gesemann/Roland Roth (Hrsg.), Handbuch Lokale Integrationspolitik, Wiesbaden 2018, S. 201-226.

Tanner, Albert u. a. (Hrsg.), Heterogenität und Integration. Umgang mit Ungleichheit und Differenz in Schule und Kindergarten, Zürich 2006.

Van Avermaet, Piet, Tests for citizenship and integration in Europe. A critical reflection, in: Barbara Haider (Hrsg.), Deutsch über alles? Sprachförderung für Erwachsene, Wien 2011, S. 59-76.

Zontini, Elisabetta, Transnational Families, Migration and Gender. Moroccan and Filipino Women in Bologna and Barcelona, New York 2010.

\section{Statistische Quellen}

Amt der Tiroler Landesregierung, Demografische Daten Tirol 2016, Innsbruck 2017, [https://www.tirol.gv.at/fileadmin/themen/statistik-budget/statistik/downloads/BEV2016. pdf], eingesehen 2. 1. 2018.

Amt der Tiroler Landesregierung, Gleichstellungsbericht Tirol. Frauen und Männer in Tirol, Wien 2016, [https://www.tirol.gv.at/fileadmin/themen/gesellschaft-soziales/frauen/downloads/Gleichstellungsbericht-2016-web.pdf], eingesehen 23. 12. 2017. 
Amt der Tiroler Landesregierung, Integration von Flüchtlingen in Tirol, o. D., [https:// www.tirol.gv.at/gesellschaft-soziales/integration/angebote-zur-integration-vonfluechtlingen-in-tirol/], eingesehen 18. 1. 2018.

Bonin, Holger, The Potential Economic Benefits of Education of Migrants in the EU. EENEE Analytical Report 31, Prepared for the European Commission, Brüssel 2017, [https:// publications.europa.eu/en/publication-detail/-/publication/223f6cbf-12b2-11e7808e-01aa75ed71a1/language-en], eingesehen 20. 1. 2018.

Bundesamt für Migration, Probleme der Integration von Ausländerinnen und Ausländern in der Schweiz. Bestandesaufnahme der Fakten, Ursachen, Risikogruppen, Maßnahmen und des integrationspolitischen Handlungsbedarfs, Bern 2006, [https://www. sem.admin.ch/dam/data/sem/integration/berichte/ber-integr-2006-d.pdf], eingesehen 2.1. 2018.

Bundesamt für Statistik, Statistischer Bericht zur Integration der Bevölkerung mit Migrationshintergrund, Neuchatêl 2004, [https://www.bfs.admin.ch/bfsstatic/dam/assets/2546310/master], eingesehen 27. 1. 2018.

Bundesministerium für Gesundheit und Frauen, Österreichischer Frauengesundheitsbericht 2010/2011, Wien 2010, [https://www.bmgf.gv.at/cms/home/attachments/1/6/5/CH1572/CMS1466495497539/frauengesundheitsbericht_2010_2011. pdf], eingesehen 28. 1. 2018.

Eurofound, Approaches to the labour market integration of refugees and asylum seekers, Publications Office of the European Union, Luxemburg 2016, [https://eeagrants. org/content/download/12903/174473/version/2/file/Eurofound+\%282016\%29+Ap proaches+to+the+labour+market+intergration+of+refugees+and+asylum+seekers. pdf], eingesehen 2. 2. 2018.

Europarat, Integration tests: helping or hindering integration? Report. Provisional version, Brüssel 2013, [http://www.assembly.coe.int/CommitteeDocs/2013/ amdoc11_2013TA.pdf], eingesehen 13. 2. 2018.

European Commission, Challenges in the labour market integration of asylum seekers and refugees, EEPO ad hoc request, Publications Office of the European Union, Luxemburg 2016, [http://ec.europa.eu/social/BlobServlet?docld=15894\&langld=en], eingesehen 20. 1. 2018.

European Commission, Employment and Social Developments in Europe. Annual Review 2016, Luxemburg 2016, [http://ec.europa.eu/social/main.jsp?catld=738\&langld= en\&publd=7952\&type=2\&furtherPubs=yes], eingesehen 2. 1. 2018.

Gesundheit Österreich GmbH, Migration und Gesundheit. Literaturbericht zur Situation in Österreich. Im Auftrag der Arbeiterkammer Wien und des Bundesministeriums für Gesundheit, Wien 2015, [https://media.arbeiterkammer.at/wien/PDF/studien/Bericht_Migration_und_Gesundheit.pdf], eingesehen 29.1. 2018. 
Internationale Organisation für Migration, Soziale Exklusion und Gesundheit von Migrant*innen in Österreich, Wien 2003, [http://www.kompass.humanrights.ch/cms/ upload/pdf/oe/gesundheit_Migrant*innen.pdf], eingesehen 2. 1. 2018.

Österreichischer Integrationsfonds, Aktuelles zu Migration und Integration. Migrant*innen in Österreich (Fact Sheet Nr. 28), Wien 2018, [https://www.integrationsfonds.at/publikationen/oeif-fact-sheets/], eingesehen 2. 2. 2018.

Pew Research Center, Spring 2016 Global Attitudes Survey. July 2016, o. O. 2016, [http:// www.pewglobal.org/files/2016/07/Pew-Research-Center-EU-Refugees-and-NationalIdentity-Report-FINAL-July-11-2016.pdf], eingesehen 22. 1. 2018.

Statistik Austria, Migration und Integration. Zahlen, Daten, Indikatoren 2017, Wien 2017, [https://www.integrationsfonds.at/fileadmin/content/AT/Downloads/Publikationen/Statistisches_Jahrbuch_migration_integration_2017.pdf], eingesehen 27. 12. 2017.

World Economic Forum, The Global Gender Gap Report 2017, Cologny-Geneva 2017, [https://www.google.at/url?sa=t\&rct=j\&q=\&esrc=s\&source=web\&cd=1\&cad=rja \&uact=8\&ved=0ahUKEwjKpvHioMjYAhWQ6KQKHS44ChEQFggtMAA\&url=https \%3A\%2F\%2Fwww.weforum.org\%2Freports\%2Fthe-global-gender-gap-report2017\&usg=AOvVaw2U005-fDAMbjpO3-LkuhWd], eingesehen 1. 1. 2018.

\section{Webseiten}

Frauen aus allen Ländern. Bildungs- und Beratungseinrichtung, Innsbruck, 2014, [https://frauenausallenlaendern.org/impressum/], eingesehen 29. 12. 2017.

Innsbrucker Verein Multikulturell, o. D., [www.migration.cc], eingesehen 2. 1. 2018.

\section{Interviewmaterial}

Volgger, Laura, Interview mit GP1, Innsbruck, 1. 2. 2018, Aufnahme bei der Verfasserin. Volgger, Laura, Interview mit GP2, Innsbruck, 13. 2. 2018, Aufnahme bei der Verfasserin.

Laura Volgger arbeitet an einer Diplomarbeit zum Thema Selbstorganisationen weiblicher Zugewanderter in Nord- und Südtirol im Rahmen des Studiums Lehramt Geschichte und Deutsch. Laura.Volgger@student.uibk.ac.at

\section{Zitation dieses Beitrages}

Laura Volgger, Migration, Integration und Isolation. Zielgruppenspezifische Fallanalyse der Lebensbereiche Familie, Bildung und Beruf weiblicher Migrantinnen in Tirol, in: historia.scribere 11 (2019), S. 87-108, [http://historia.scribere.at], eingesehen 17.6.2019 (=aktuelles Datum).

(C) Creative Commons Licences 3.0 Österreich unter Wahrung der Urheberrechte der Autorlnnen. 
